\title{
Numerical simulations of elliptical galaxies
}

\author{
Chiaki Kobayashi ${ }^{1}$ \\ ${ }^{1}$ The Australian National University, Mt. Stromlo Observatory, Cotter Rd., Weston ACT \\ 2611, email: chiaki@mso.anu.edu.au
}

For the formation of elliptical galaxies, two scenarios, monolithic collapse vs. major merger, have been debated. We simulate the formation and chemodynamical evolution of 128 Es from the CDM initial fluctuations, using the GRAPE-SPH code that include star formation, supernovae feedback, and chemical enrichment. In our CDM-based scenario, galaxies form through the successive merging of subgalaxies with various masses.

The metallicity gradient gives one of the most stringent constraints on the galaxy formation. Kobayashi (2004) showed that gradients are destroyed by mergers and are not enough regenerated by induced star formation. In observations, there is a significant scatter (Kobayashi \& Arimoto 1999), which is reproduced with our simulations (Fig. 1a). The scatter stems from the difference in the merging histories. Galaxies that form monolithically have steeper gradients, while galaxies that undergo major mergers have shallower gradients. For less-massive galaxies, Spolaor et al. (2009) showed that the gradients become flatter, which cannot be reproduced.

This scenario does not conflict with the observed scaling relations. The mass-metallicity relation (Fig. 1b) and the fundamental plane are also reproduced (Kobayashi 2005). An intrinsic scatter exists along the fundamental plane, and the origin of this scatter lies in differences in merging history. Galaxies that undergo major mergers tend to have larger effective radii $r_{\mathrm{e}}$ and fainter surface brightnesses, which result in larger masses, smaller surface brightnesses, and larger mass-to-light ratios $M / L$.

Mass-to-light ratios and baryon fractions (Fig. 1c) will be important constraints on the origin of elliptical galaxies. Our simulations are roughly consistent with the available observations with X-ray (Nagino \& Matsushita 2009), optical measurements (Gerhard et al. 2001), and gravitational lensing (Treu et al. 2006). The stellar $M / L$ correlates with the stellar mass, which can be the origin of the tilt of the fundamental plane. The total $M / L$ does not correlate with the stellar $M / L$, which is contrary to the optical observations. The total $M / L$ are almost constant as $\sim 18-28$ in $2 r_{\mathrm{e}}$ for $M_{*} \gtrsim 10^{10} M_{\odot}$, which is consistent with the X-ray observations, and increases upto $\sim 100$ for dwarf ellipticals. Thus, the baryon fraction is larger for massive galaxies.
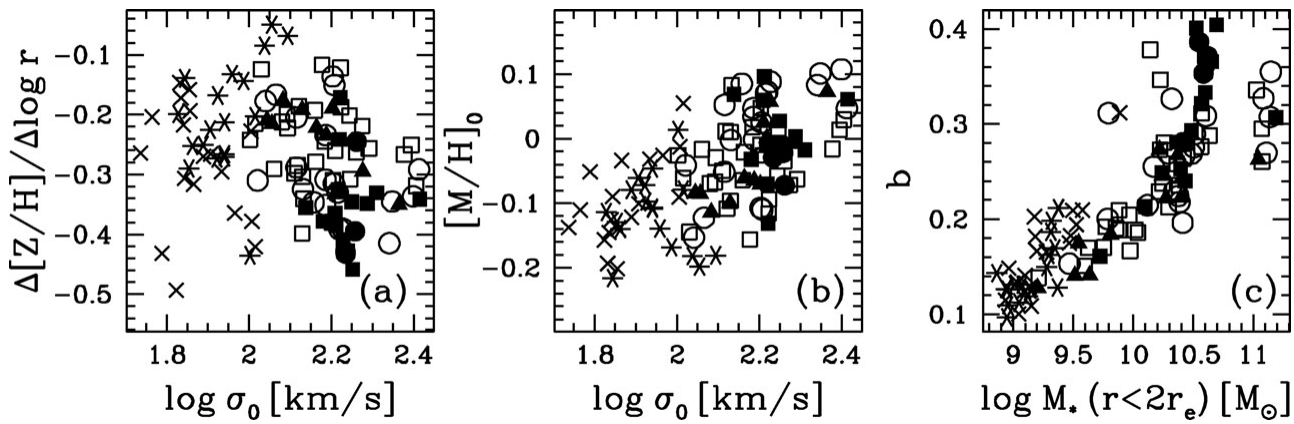

Figure 1. (a) Metallicity gradients and (b) central metallicities against central velocity dispersions for monolithic-like (filled symbols), major merger (open symbols), and dwarf ellipticals (crosses). (c) Baryon fractions against stellar mass within $2 r_{\mathrm{e}}$ of the simulated galaxies. 